\title{
Norois
}

Environnement, aménagement, société

\section{Pratiques agricoles, perceptions et représentations du paysage : quelles articulations ? Approches croisées Nord/Sud}

Agricultural practices, landscape perceptions and representations: what kind of links? North/South crossing approaches

\section{Sébastien Caillault et Maxime Marie}

\section{OpenEdition \\ Journals}

Édition électronique

URL : https://journals.openedition.org/norois/2995

DOI : 10.4000/norois.2995

ISBN : 978-2-7535-1561-1

ISSN : 1760-8546

Éditeur

Presses universitaires de Rennes

\section{Édition imprimée}

Date de publication : 15 décembre 2009

Pagination : $9-20$

ISBN : 978-2-7535-1050-0

ISSN : 0029-182X

Référence électronique

Sébastien Caillault et Maxime Marie, «Pratiques agricoles, perceptions et représentations du paysage : quelles articulations? Approches croisées Nord/Sud », Norois [En ligne], 213 | 2009/4, mis en ligne le 15 décembre 2011, consulté le 14 janvier 2022. URL : http://journals.openedition.org/ norois/2995 ; DOI : https://doi.org/10.4000/norois.2995 


\title{
Pratiques agricoles, perceptions et représentations du paysage : QUelles ARTiculations? Approches Croisées Nord/Sud
}

\author{
Sébastien Caillault, Maxime Marie \\ UMR 6554 CNRS - LETG Géophen \\ (Université de Caen Basse-Normandie), \\ Esplanade de la Paix - 14032 CaEn cedex \\ sebastien.caillault@unicaen.fr,maxime.marie@unicaen.fr
}

\section{RÉSUMÉ}

Cet article propose d'exposer un point de vue sur le paysage en tant que " production du quotidien». En partant de l'exemple des espaces agricoles, nous tentons une réflexion sur les articulations entre les dimensions matérielles et idéelles de la production de paysage en géographie. L'objectif de cette démarche est de comprendre les dynamiques observables dans des espaces agricoles variés (Afrique de l'Ouest, Basse-Normandie, Galice et Sud de l'Angleterre). Nous nous attacherons d'abord à étudier la production de paysage vue comme le résultat de pratiques individuelles répondant à des logiques quotidiennes correspondant à des pratiques "utiles » mais aussi à des pratiques " agréables ». Sous cet angle, analyser la perception d'un objet par un individu permet de mieux comprendre ses actions sur ce même objet. Par ailleurs, les pratiques individuelles sont aussi régies par des logiques collectives liées en partie à des processus d'identification sociale et culturelle qui définissent le sens que les individus donnent à ces pratiques. C'est pourquoi, nous proposerons ensuite une réflexion sur l'importance des manières de concevoir une chose (un métier ou une activité, un paysage...) pour les individus au sein d'un groupe social et sur les implications de ces conceptions sur la mise en ouvre des pratiques agricoles. Le "groupe » des agriculteurs produit ainsi des représentations sociales qui définissent des types de comportements singuliers par rapport à l'espace et au paysage. À partir d'exemples variés, issus de nos différents travaux de recherche en cours, nous essaierons de proposer des clés de lecture communes pour analyser la production tangible de paysage à travers les échelles et la complexité des contextes locaux (espace/temps/société).

MOTS CLÉ : production de paysage - pratiques agricoles - rationalité - perceptions de l'espace - représentations sociales

\section{ABSTRACT \\ Agricultural practices, landscape perceptions and representations: what kind of links? North/South crossing approaches}

This paper tries to expose a point view of the landscape as "an everyday (daily) production". Based on examples of agricultural land, we try a geographical reflection on the linkages between material and ideal dimensions of landscape production in geography. The objective of this approach is to understand dynamics observed in various agricultural areas (West Africa, Lower Normandy, Galicia and Southern England). We will first strive to study the production of landscape as the result of individual practices responding to process corresponding to daily practices "useful" but also "pleasant" practices. From this angle, ana- 
lyzing the perception of an object by a person allows a better understanding of its actions on this object. In addition, individual practices are also governed by collective processes related processes of social and cultural identification that define the meaning that individuals give to their practices. That's why, we then propose a reflection on the importance of ways to describe something (a job, an activity or a landscape...) for individuals within a social group and the implications of these conceptions on agricultural practices. The "group" of farmers produces social representations that define types of singular behaviour in relation to space and landscape. From various examples, from our ongoing research, we will try to propose common key reading to analyze the production of tangible landscape through scales and complexity of local contexts (space/time/society).

KEY WORDS : landscape production - agricultural practices - rationality - space perceptions - social representations

L'agriculture apparaît bien souvent comme une activité qui contribue à la production de paysage dans la mesure où, elle aménage l'espace, le structure et le transforme. Les paysages agricoles, dans leurs dimensions matérielles, peuvent ainsi être abordés comme le résultat du fonctionnement des systèmes agricoles dans l'espace (Benoît, 1990; Poinsot, 2008). De nombreux travaux de géographes, d'agronomes ou d'écologues font explicitement référence à une approche des structures des paysages par l'analyse de l'organisation technico-économique des systèmes agricoles (Deffontaines, 2001 ; Thenail, 1996 ; Serpantié et al., 2000). Dans cette grille de lecture on sous-tend l'idée que les mutations de l'agriculture expliquent, au moins en partie, les dynamiques de transformation des paysages ruraux, et ce, d'une échelle locale à régionale (Houet et al., 2008; Pourtier, 2003).

Les équilibres qui régissent la production de paysage par l'agriculture sont néanmoins dépendants de nombreux facteurs dont la hiérarchie peut varier considérablement selon les contextes locaux (paramètres pédoclimatiques, orientations des systèmes de production, niveau maîtrise technique, structures foncières, niveau d'intégration économique, politiques publiques, organisation des rapports sociaux...). Malgré cette variabilité et pour dépasser une vision (trop) déterministe, la compréhension de cette production paysagère par l'agriculture ne peut s'affranchir d'une analyse fine des pratiques des agriculteurs. C'est en effet en s'intéressant au niveau le plus élémentaire de la production de paysage, ici les pratiques agricoles, que l'on peut tenter de dégager des logiques communes à différents contextes régionaux (Deffontaines, 1996). Dans cette optique, une première phase consiste souvent à analyser les formes du paysage, leur localisation et leur agencement.

Cette phase d'analyse spatiale réalisée, il advient alors de comprendre quelles sont les fonctions qui participent à la production de ces formes et donc à définir le paysage-objet. Seulement, si la dimension matérielle du paysage reste relativement simple à isoler pour le chercheur (paysageobjet), les logiques de la production de ce «paysage-objet » le sont beaucoup moins (Deffontaines, 2004), essentiellement dans la mesure où elles relèvent de la relation dialectique entre une dimension matérielle et une dimension représentée du paysage. Ainsi, pour illustrer cette idée on peut reprendre et adapter une citation d'A. Berque (1992) : «Les agriculteurs perçoivent l'espace en fonction des aménagements qu'ils en font et l'aménagent en fonctions des perceptions qu'ils en ont. » L'enjeu d'une telle approche peut être, par exemple, de construire des modèles explicatifs généraux des différentes modalités de production de paysage par les activités agricoles.

Cette démarche place alors les agriculteurs et leurs actions au centre d'un système multi scalaire dans lequel s'articulent à la fois les dimensions matérielles et les perceptions du paysage, mais aussi les dimensions individuelles et collectives de leurs pratiques (Landais et al., 1988). Elle permet aussi de mettre en évidence des mécanismes communs dans des contextes culturels et socio-économiques différents. 
Cet article est le fruit de la confrontation des résultats de deux travaux de recherches sur la production de paysage. Ces deux recherches ont pour terrains des espaces très différents : l'ouest du Burkina Faso pour la première, et les bocages de l'ouest européens pour la seconde (Galice, BasseNormandie et Sud de l'Angleterre). C'est pourquoi, il convient de préciser quelques définitions afin de bien circonscrire le champ thématique et ainsi permettre la comparaison des mécanismes de production de paysage dans des espaces aussi variés.

Tout d'abord, l'analyse des pratiques est vue à travers le système de rationalité de l'agriculteur et des ses raisons d'agir (Darré et al., 2004). Pour comprendre ces comportements il convient donc d'interroger les individus sur la manière dont ils perçoivent la réalité et sur la signification qu'ils donnent à leurs pratiques (Godelier, 1984; Deffontaines et Petit, 1985; Soulard, 1999). De cette manière, les pratiques individuelles peuvent être analysées comme des comportements répondant aux logiques du quotidien des individus reconnaissant alors la production du paysage comme le résultat de gestes quotidiens (Friedberg, 1992; Rossi, 2000), observée à un moment donné.

À partir des interrogations sur la construction quotidienne des paysages, nous nous sommes alors plus concentrés sur les pratiques s'exprimant dans un temps cours. Les logiques telles que les notions d'héritage ou de cycle de vie d'exploitation ne sont pas abordées dans ce texte. Chez les agriculteurs, ces logiques du quotidien confèrent aux pratiques agricoles plusieurs dimensions imbriquées : une dimension " utile » (dépendant de finalités productives), une dimension «sociale » (répondant à des normes de comportement), et une dimension " agréable ( dépendant d'un système de valeur). Le parti pris est ici de considérer que ces trois dimensions des pratiques ne sont jamais dissociables les unes des autres et qu'elles participent chacune à la construction des deux autres. L'importance de telle ou telle dimension peut cependant varier de façon considérable selon les types de pratiques, les individus et les contextes dans lesquels elles sont mises en œuvre (Darré, 1985).

Quelles sont alors les articulations entre ces dimensions et qu'apporte leur compréhension à l'étude des mécanismes de production de paysage?

La première partie de cet article met en lumière les pré-requis qui doivent être considérés pour l'étude des espaces et des paysages agricoles (dimension fonctionnelle). Dans la deuxième partie, les auteurs cherchent à comprendre la dialectique qui s'établit, à l'échelle individuelle, entre les pratiques des agriculteurs et la perception qu'ils ont de leur environnement direct (espace et paysage). Dans la troisième partie c'est l'articulation entre, la dimension individuelle des pratiques et des perceptions, et la dimension collective des représentations qui est interrogée. Enfin, dans la dernière partie de l'article les auteurs proposent un schéma explicitant les liens et les articulations entre les différentes dimensions des pratiques en fournissant un cadre général d'analyse de la production de paysage par les activités agricoles.

\section{L'analyse spatiale comme préalable à l'étude des systèmes agricoles}

La définition du paysage-objet dans des espaces ruraux peut se faire par l'étude de la morphologie agraire (par exemple, les formes de parcellaire). Elle renseigne alors sur les moyens mis en œuvre pour utiliser la terre à des fins productives, et ce, suivant des potentiels agro-pédologiques ou bien des règles d'organisation foncière variables dans le temps et l'espace.

Au Burkina Faso par exemple (fig. 1 - planche I), l'analyse des formes de champs dans un espace donné nous permet de comprendre l'importance de la mécanisation à travers la proportion des grands blocs de cultures « rectangulaires » (culture attelée, tracteur...) se détachant des petites parcelles cultivées manuellement. Cette analyse nous révèle alors la transformation d'un système de production qui est passé d'une fonction majoritairement vivrière (exploitations de grandes familles) à une fonction désormais marchande (exploitations individualisées) à travers les cultures de coton, de maïs ou de riz (Tersiguel, 1995; Tallet, 2007).

Une autre illustration de l'analyse spatiale est l'étude des structures d'exploitations agricoles. L'analyse de la répartition des parcelles et de leur rotation dans les systèmes laitiers dans le Sud de 
l'Angleterre met en évidence un modèle d'organisation « centre - périphérie » (Marie et Delahaye, 2009) (fig. 2 - planche II). Dans ces exploitations laitières, les parcelles les plus proches du siège se trouvent souvent vouées au pâturage des vaches laitières. En s'éloignant du siège, les cultures fourragères deviennent de plus en plus fréquentes (maïs fourrage). Enfin, lorsque des parcelles sont très éloignées, les agriculteurs les conduisent soit en céréales (grandes parcelles) soit en herbe sur un mode «extensif » (petites parcelles), afin de minimiser le nombre d'interventions sur ces parcelles (pâture des génisses par exemple). Dans cet exemple, l'analyse de l'organisation spatiale des exploitations peut se faire suivant la théorie des comportements rationnels, et ce, en faisant intervenir des paramètres simples tels que la distance, la surface et l'accessibilité des parcelles.

Au final, l'analyse spatiale est alors vue comme un préalable intéressant. Elle permet en effet d'appréhender les modèles d'organisation agricole en jeu mais aussi de fournir aux lecteurs extérieurs un cadre objectivé pour l'appréhension des espaces étudiés. Cette phase d'analyse par superposition et croisement de variables localisées est intéressante (et désormais simple à mettre en œuvre avec les SIG) mais ne peut permettre la compréhension des mécanismes produisant le paysage - objet.

Si ces «modèles » semblent globalement valides, ils ne sont néanmoins pas dénués de connotation réduisant le réel à des comportements qui se suivent dans une conception linéaire du temps (exemple du processus de «modernisation » Burkina Faso) ou bien à des comportements guidés par les lois euclidiennes (exemple de l'importance des distances dans les exploitations laitières). Pourtant le paysage est produit par les comportements et les actions des individus dans un espace donné. Dans le temps, l'approche fonctionnelle réduit l'histoire à un processus où les choix se suivent de manière logique en omettant l'ensemble des autres options qui auraient pu être possibles. Dans l'espace, les fonctions attribuées aux parcelles sont en parties guidées par la distance au siège et l'investissement « travail » (vu comme un coût). Si le modèle est valide il ne doit faire oublier que les agriculteurs ne font pas ces choix de traitement sur leurs parcelles par un calcul de l'optimalité coût/distance. Les actions des individus ne s'orientent donc pas qu'en fonction d'un optimum à atteindre (Simon, 1969). La construction de modèles dans cette visée est alors un cadre de départ mais il est important d'en voir les limites.

\section{Quelles dialectiques entre pratiques agricoles et perceptions du paysage par les agriculteurs?}

Dans l'hypothèse que les comportements produisent le paysage objet que nous observons, il paraît nécessaire de comprendre les pratiques mises en place. Généralement les liens entre contexte agricole régional et pratiques sont envisagés de manière simple. Ils montrent alors les productions régionales et les usages associés (calendriers des pratiques, par exemple). Cette visée permet d'analyser les paysages régionaux et les usages qui y sont pratiqués pour un contexte socioéconomique donné.

Comprendre le sens de la pratique en un lieu donné ne peut se contenter de cette seule lecture utilitariste. Il semble alors nécessaire de prendre en compte les systèmes de rationalité des individus. Ces systèmes de rationalité sont construits en partie par les rapports qu'entretiennent les individus avec la réalité locale, et ce, qu'elle soit liée au contexte économique, social, culturel ou naturel. Ces derniers permettent alors d'aborder les usages et les pratiques par l'individu. Ainsi, de nombreux exemples montrent que les usages de l'espace sont indissociables des perceptions que les individus ont de l'espace et des objets associés aux usages qu'ils font de cet espace.

Cette interaction usage/perception a par exemple été étudiée par quelques chercheurs à travers les classifications empiriques des sols utilisées par les agriculteurs (Blanc-Pamard et Millevile, 1985; Veauvy, 1991). En effet, pour un type de sol donné, une culture préférentielle et une action sont souvent pensées. Au delà de la correspondance entre la catégorisation empirique et la catégorisation scientifique des objets naturels (Fairhead et Leach, 1996), ces aspects interrogent 
les manières de percevoir le sol pour aboutir à des systèmes de classification empiriques (non intentionnel) guidant les actions des agriculteurs.

Suivant quels principes ces « classifications » sont-elles construites par les agriculteurs? Il y a évidemment les qualités du sol (structure/texture/couleur) mais aussi la pensée des gestes possibles sur cette terre (l'usage). On remarque ainsi que les terres limoneuses ou sableuses sont systématiquement classées parmi les «bonnes terres », et définies comme des terres « légères » ou « facile à travailler ». Ces classifications n'obéissent pas réellement aux logiques et aux catégories d'analyse des pédologues mais davantage à la relation dialectique perception/expérience qui s'établit au quotidien à travers les pratiques de l'agriculteur (Soulard, 1999; Marie, 2008). Ainsi, plus que le type de sol (défini scientifiquement par le pédologue), c'est la «terre » (et ses qualités associées) telle qu'elle est perçue par l'agriculteur qui peut permettre de mieux comprendre l'organisation des pratiques dans l'espace. Dans le cas des exploitations d'élevage bovin en Normandie, par exemple, l'agencement spatial des fonctions parcellaires apparaît comme étant le résultat de combinaisons complexes entre facteurs d'organisation où les phénomènes de perception de l'espace occupent des places variables selon les types de système de production et le niveau de contrainte induit par les propriétés des sols de l'exploitation - essentiellement hydriques et mécaniques.

De plus, le rapport des agriculteurs au sol révèle une autre dimension de leurs pratiques. En effet, parallèlement à la dimension utilitariste des pratiques, qui dépend de l'objectif défini au sein du système de rationalité de l'individu, une autre dimension apparaît lorsque l'on prend la peine d'analyser le discours des agriculteurs sur leurs pratiques. La notion de plaisir, c'est-à-dire la dimension agréable du geste, est souvent sous-jacente dans les paroles des agriculteurs. La pratique du labour revêt ainsi souvent une connotation très positive dans l'esprit des agriculteurs, en partie liée à l'utilisation de machines puissantes et à l'idée de contrôle, voire de domination de la nature (Prével, 2007). Dans cette optique, la terre est ainsi souvent perçue à travers les aspects agréables du travail du sol, de nombreux agriculteurs définissent ainsi certains sols en fonction du plaisir qu'ils trouvent à les labourer (photo 1).

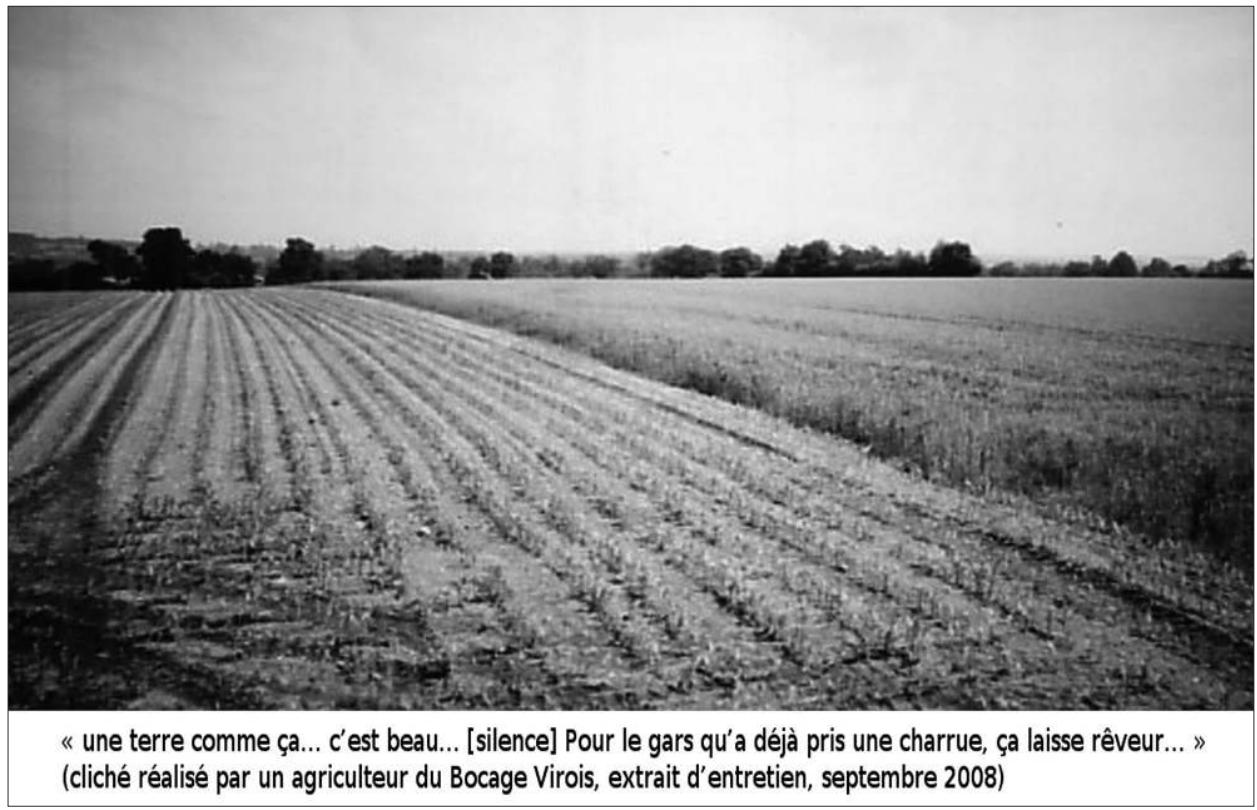

Photo 1 : La dimension agréable des pratiques Pleasant dimension of practices 
L'importance du geste, du plaisir qui peut s'en dégager construit donc aussi cette « classification ». « Les gestes utiles ne doivent pas faire oublier les gestes agréables » écrivait G. Bachelard (1938), il ne faut pas le voir comme deux gestes séparés, bien au contraire, cela pousse à comprendre en quoi au quotidien l'utile et l'agréable construisent des pratiques produisant du paysage.

La production de paysage, étudiée suivant une approche compréhensive, permet alors d'appréhender plus facilement les raisons des pratiques. Les pratiques s'inscrivent ainsi dans un espace borné de choix (commun à tous les individus : contexte socio-économique, débouchés des denrées) mêlé à une dimension personnelle où chaque individu agit sur l'espace en fonction de ses conceptions (travail, plaisir du geste, esthétique de la parcelle...).

Cette idée permet donc de sortir d'une vision seulement utilitariste qui ne permet pas toujours une compréhension des pratiques pourtant essentielle à la lecture des paysages agricoles. Ainsi, en Afrique de l'Ouest, l'observation des feux (photo 2) dans certains espaces ne peut se comprendre à la seule lecture utilitariste de pratiques comme la chasse, l'ouverture de la brousse... Certaines portions de l'espace ne correspondent pas à ces usages (espace trop petit, sans intérêt d'ouverture...) pourtant le feu y est pratiqué. La seule vision utilitariste nous place alors dans une impasse. Pourtant des raisons expliquent la présence du feu car il est présent chaque année sur les mêmes lieux. Pour chercher à comprendre ces "raisons du feu », il parait souhaitable de renverser la question. D'une question utilitariste : "à quoi servent ces feux? " nous pouvons envisager une question qui interroge la pratique même du feu «que représente le feu pour les individus? ». Comment se construisent alors ces représentations du feu et n'y a-t-il que du plaisir à mettre le feu?

Photo 2 : Feux de brousse sous parcs arborés dans l'ouest burkinabé.

in western Burkina Faso

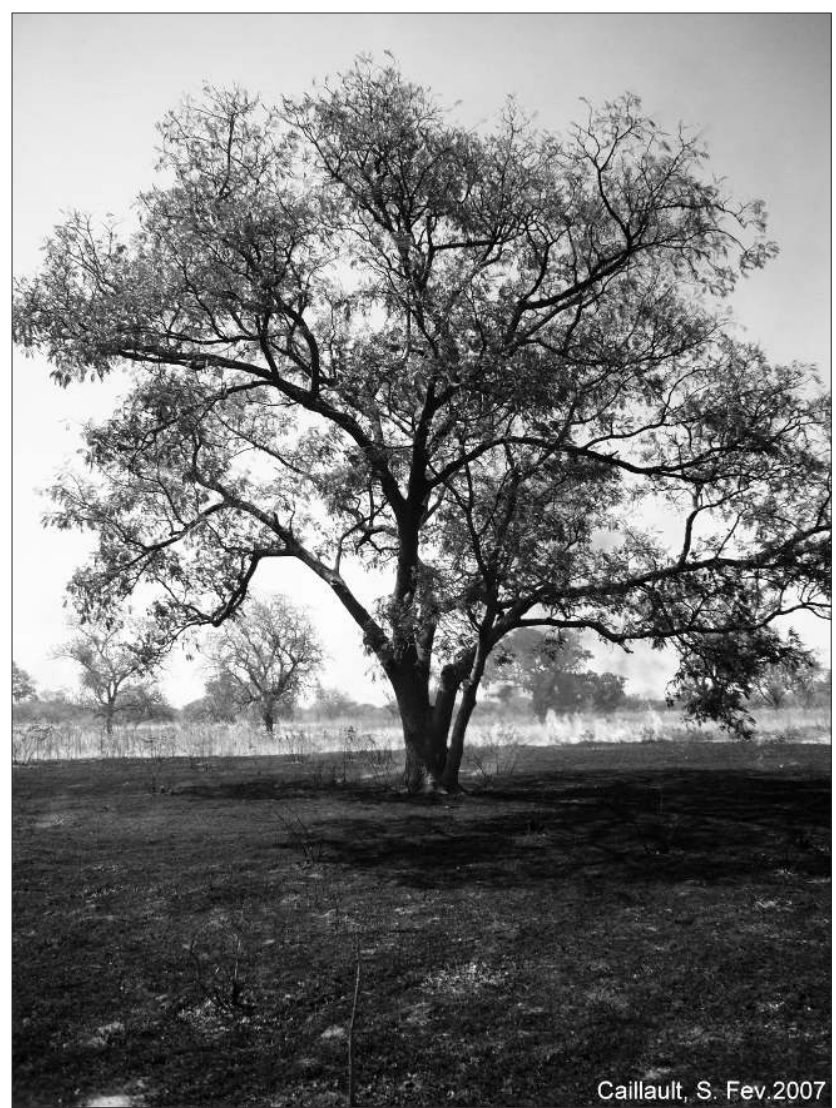




\section{De l'expression des dynamiques collectives aux représentations sociales}

Si ce plaisir du feu ou le plaisir de passer la charrue existe, ces gestes répondent à des référentiels de l'utile et de l'agréable. La construction de ces référentiels nous interroge alors sur la dimension collective des gestes du quotidien. En effet, certaines pratiques recouvrent des valeurs différentes au sein d'un groupe social suivant les représentations sociales auxquelles elles renvoient (Le Floch et al., 2005).

Pour le cas du feu dans certaines populations, les feux individuels dans la brousse sont interdits par l'autorité coutumière. Cet interdit est levé lors d'un incendie rituel qui concerne l'ensemble des individus du village, cérémonie pendant laquelle le feu est mis. Ensuite un ramassage des animaux est effectué (rats, criquets...) (Dugast, 2008). C'est seulement après cette cérémonie de « délivrance » que les feux individuels peuvent alors être admis sur le terroir villageois. On comprend donc comment le collectif peut participer à construire le plaisir individuel qui ne devient possible qu'après et avec l'attente de la cérémonie collective.

En Normandie, cette dimension collective est aussi présente dans la manière dont les agriculteurs conçoivent leur métier et la place qu'il doit occuper dans la société française. Ainsi, la prise en compte des différents systèmes de valeurs existant au sein du monde agricole permet de comprendre comment les pratiques des agriculteurs s'inscrivent dans des modèles de références et de normes de comportement (Peyre, 2004).

Le cas des représentations paysagères des agriculteurs semble ainsi intéressant pour illustrer la prégnance de ces modèles et de ces normes. En effet, les agriculteurs de Normandie, associent des objets du paysage à des valeurs souvent communes à l'ensemble du monde agricole actuel. Ces représentations révèlent alors une conception vue « de l'intérieur » d'un objet perçu dans un contexte social et démographique donné. C'est ce que montre une enquête réalisée auprès d'une vingtaine d'exploitants agricoles en Normandie. Cette dernière a consisté à distribuer des appareils photos jetables aux exploitants pour qu'ils réalisent des clichés servant ensuite de support aux entretiens réalisés avec eux (Michelin, 1998; Marie, 2008).

La friche par exemple (fig. 3, photo 1 et 2 - planche II) cristallise chez une grande partie des exploitants, le rejet et les sentiments négatifs à l'égard du paysage. Cet élément du paysage-objet, est souvent associé au recul des activités agricoles dans l'occupation de l'espace et à la baisse du nombre d'agriculteurs. Un agriculteur évoque la friche en ces termes lorsqu'il examine la photo 1 : «Les endroits comme ça, font peine à voir, on se dit que c'est triste... y a plus de vie... » (Cliché réalisé par un agriculteur du Bocage Virois, extrait d'entretien, septembre 2008).

Dans les discours des agriculteurs, la friche est également souvent associée à la notion de travail, un agriculteur du Pays d'Auge aborde cette notion en observant un cliché qu'il a lui-même réalisé : «Souvent sur les buttes ça s'encrasse... Alors, bon, si le gars il est courageux, ça va, y nettoie, mais... si il sait pas bosser, ça reste comme ça » (extrait d'entretien, février 2006).

Ces éléments renvoient à la place des activités agricoles dans les espaces ruraux, surtout en Normandie où la surface agricole utile occupe souvent plus de $80 \%$ du territoire. Les agriculteurs normands fortement marqués par l'idéologie du progrès et de la modernisation agricole, refusent en effet toute idée de déprise ou de recul de l'emprise de l'agriculture dans l'espace. Les friches sont pour eux, des éléments symptomatiques de la diminution du nombre d'exploitations.

Cependant, la friche est aussi souvent perçue comme le révélateur des transformations du métier d'agriculteur : augmentation de la productivité mais aussi de la charge de travail. Celui qui n'arrive plus à faire face à cette charge de travail est alors stigmatisé comme un «fainéant ». Le rapport au travail, la capacité à travailler et à produire toujours davantage, est en effet central (Prével, 2007). Pour les agriculteurs, les éléments du paysage portent donc différentes valeurs symboliques liées aux représentations qu'ils ont de leur propre métier.

Au contraire, lorsque les exploitants agricoles évoquent les éléments associés à la culture du maïs fourrage ou des céréales (grandes parcelles aux formes simples), les discours recouvrent une dimension beaucoup plus positive (Guisepelli, 2005), un agriculteur du Sud Manche nous dit par 
exemple (fig. 3, photo 3 - planche II) : «là au moins c'est propre, c'est net. Et puis, y a pas de pointe et $y$ a de la place... enfin, j'veux dire, c'est pas trop petit... c'est plus moderne quoi... pour le travail » (cliché réalisé par l'agriculteur, extrait d'entretien, mars 2006). Un autre, dans le Bocage Virois confie que «[les cultures] ça donne une image dynamique des exploitations, parce que bon quand on voit des cultures bien menées, on se dit que les agriculteurs y sont au point... niveau technique et niveau boulot» (photo 4, réalisé par l'agriculteur, extrait d'entretien, septembre 2008).

La compréhension de la place du travail dans les représentations qu’ont les agriculteurs de leur métier apparaît donc comme essentielle pour analyser les pratiques de ces derniers (B. Lémery, 1986 ; S. Calvo-Iglesias et al., 2006).

L'exemple de la culture du mil dans une zone de migration du Burkina Faso (bassin cotonnier Ouest) illustre aussi cette articulation de l'utile et de l'agréable en fonction de normes différenciées. En effet, la culture du « petit mil » dans cette zone cotonnière est souvent le fait de jeunes agriculteurs Mossi. Ces derniers venant de zones plus sèches préfèrent souvent cette céréale aux autres notamment pour la question du goût du thô produit.

À l'inverse, d'autres perçoivent cette céréale de manière différente. Ainsi, un jeune agriculteur Bwa (ethnie autochtone) dans son champ de maïs, nous indique de manière explicite la localisation et sa vision négative à l'égard des champs de mil: "le petit mil c'est un travail difficile [épines coupantes sur la tige], c'est dans le bas avec les Mossi » (extrait d'entretien, novembre 2008).

Nous voyons au final comment le pénible ou l'agréable produisent du paysage par le biais d'usages aux dimensions qui varient en fonction de groupes mobilisant des référentiels différents.

Ces représentations peuvent être produites localement au sein des réseaux de sociabilité des agriculteurs (voisinage, CUMA...), mais peuvent également l'être à des niveaux plus importants par les organisations professionnelles agricoles, les industries agro-chimiques, ou les médias (fig. 4, doc. 1).

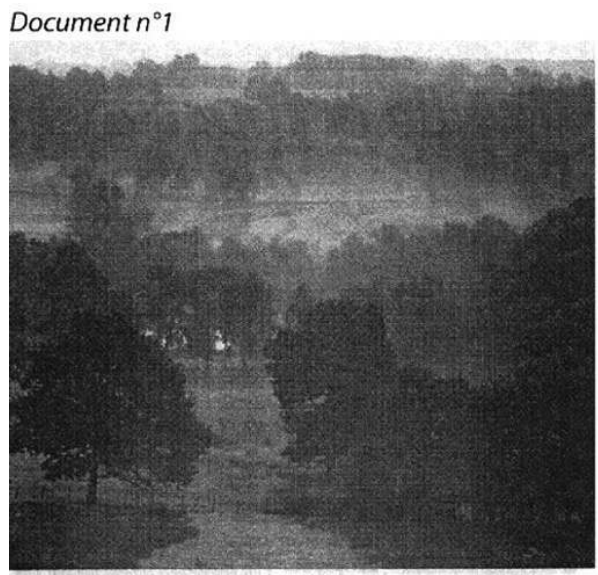

C'est beau une région sans broussaille!

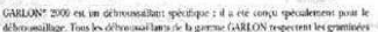

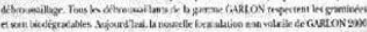

\section{GARLONN}

\section{Document $n^{\circ} 2$}

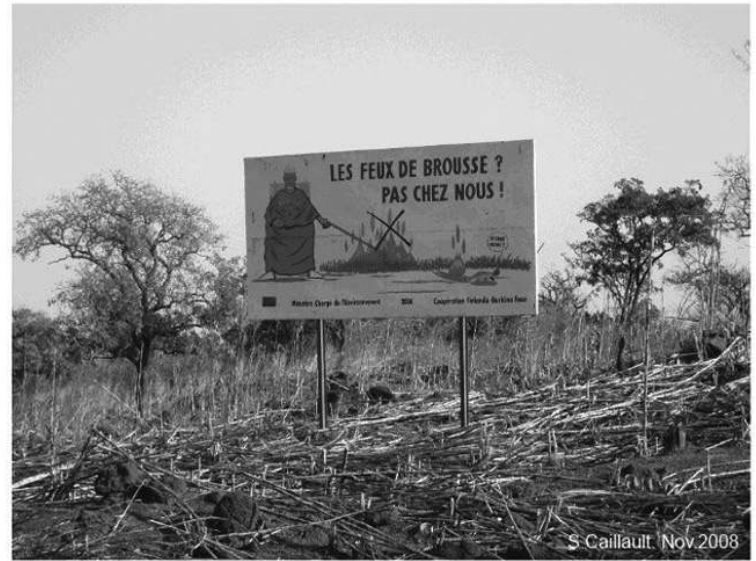

Panneau de sensibilisation à l'environnement Agence de coopération Finlande / Burkina Faso. "Les feux de brousse? Pas chez nous!"

(in M.Prével, 2007)

Photo 2 : Feux de brousse sous parcs arborés dans l'ouest burkinabé. Bush fires in wooded parks in western Burkina Faso 
Dans les sociétés rurales d'Afrique de l'Ouest, on observe une situation du même type en ce qui concerne la modernisation agricole ou bien les préoccupations environnementales. Les réseaux de sociabilité villageoise y jouent évidement un rôle puissant, mais aussi certaines firmes, ONG ou l'État occupent également une place importante dans la co-construction des représentations des individus. C'est notamment le cas pour les usages du feu dans les gestions « locales », où sous couvert de "gouvernance » et à travers des méthodes de sensibilisation (Bassett et al., 2007), le feu est souvent décrit aux populations rurales comme une pratique dépassée. Ainsi, alors que le feu est une pratique qui est avérée depuis des siècles dans les zones soudaniennes d'Afrique de l'Ouest (Ballouche, 2002), différentes ONG condamnent son usage lors de campagnes de communication locales (fig. 4, doc. 2). Ces faits interrogent alors la capacité des populations rurales à recevoir, à intégrer et à critiquer cette «bonne pensée » face à leur expérience du quotidien (De Sartre et al., 2005).

\section{Articuler les dimensions de la production de paysage : essai de formalisation}

Les représentations qu'ont les individus du paysage, et à travers elles, les manières qu'ils ont de concevoir un métier ou une pratique, relèvent donc en partie d'une construction collective. Les conceptions de l'utile, du beau ou de l'agréable ne sont pas universelles, elles trouvent leurs origines dans des trajectoires de vie et des processus d'identification à un groupe social et à une part de ses valeurs (Darré et al., 2004). C'est pourquoi il convient de prendre en compte les contextes de socialisation des individus pour comprendre leurs rapports aux objets du paysage et aux pratiques qui les produisent. Ces éléments font écho aux interrogations soulevées par Denise Pumain (2003) à propos de la complexité des processus régissant la production d'entités géographiques comme le sont les paysages. Elle souligne bien l'intérêt de questionner l'articulation des différents niveaux d'organisation sociale qui génèrent ces entités, et de la place des individus dans ce processus.

Cette articulation des échelles individuelle et collective de production de représentations et de paysage matériel se heurte cependant à un problème théorique et épistémologique majeur. En effet, comme l'écrit D. Pumain : «Aujourd'hui encore, lorsqu'il s'agit de comprendre et de formaliser l'émergence d'entités géographiques collectives, la théorie ne permet que rarement de relier explicitement les niveaux d'action et de représentations des individus et celui des entités géographiques qu'ils construisent » (Pumain, 2003, p. 26).

Dans cette optique, nous proposons un schéma des pratiques centré sur un «système agriculteur-exploitation-paysage » (fig. 5 - planche III). Celui-ci se décline autour de trois dimensions : utile, sociale, perception/action. Dans ce schéma se croisent à la fois un axe rationnel, celui des pratiques (dimension utile, en noir sur le schéma), les aspects relatifs aux interactions entre les perceptions, les représentations et les usages (dimension perception / action, en rouge) et enfin la sphère de socialisation liée aux réseaux d'interconnaissance et d'échanges des agriculteurs (dimension sociale, en orange).

Ces trois dimensions imbriquées s’intègrent alors à un niveau supérieur, celui du contexte de production, notamment à travers les circuits de commercialisation des produits agricoles mais aussi les politiques publiques mises à petite échelle (en gris sur le schéma, cf. mesures de soutien à l'agriculture : Politique Agricole Commune en Europe, Programme National de Gestion des Terroirs au Burkina Faso...). Les mécanismes évoqués ici se retrouvent dans les trois dimensions de ce schéma. Ce dernier permet ainsi d'appréhender les articulations entre les pratiques et leurs déterminants d'une part, et, la production tangible de paysage d'autre part. Cette approche, mise en place pour étudier les pratiques des agriculteurs sur des terrains variés, parait donc une piste intéressante pour l'analyse des liens entre les pratiques, les perceptions et les représentations du paysage. 


\section{Conclusion}

À travers les éléments développés dans ce texte, les différentes dimensions des pratiques apparaissent comme essentielles pour comprendre la production de paysage. La prise en compte de la dimension utile des pratiques (construite au sein du système de rationalité des individus) et de la dimension agréable de ces dernières (le sens et la valeur que les individus donnent à leurs actions) permet de mettre en évidence les ressorts de la production individuelle de paysage par les agriculteurs. De plus, le liens entre les processus de construction des représentations et de normes sociales (référentiels de comportement, représentations du paysage...) au sein des réseaux de sociabilité agricoles et les manières qu’ont les agriculteurs de concevoir leurs pratiques (utilité, plaisir), permet de mieux comprendre comment s'articulent les échelles individuelles et collectives de la production de paysage.

Ce texte évoque peu le thème des politiques publiques car il nous a semblé essentiel d'interroger en premier lieu l'étendue de «l'espace des choix » des individus (en fonction de leurs conditions matérielles d'existence et des normes de comportement auxquelles ils sont contraints). Cette approche centrée sur les logiques du « quotidien », celles qui motivent concrètement les pratiques des agriculteurs, permet de ne pas se cloisonner à une approche régionaliste et permet ainsi de tenter une formalisation conceptuelle de la production de paysage tel qu'elle peut être entendue en géographie (N. Mathieu, 1992).

\section{Remerciements}

Nous tenons à remercier les agriculteurs normands, anglais et burkinabés pour leur gentillesse et leur disponibilité. Nous souhaitons également remercier les évaluateurs, l'équipe de la revue Norois et des premières journées doctorales du paysage, pour l'attention et l'intérêt qu'ils ont manifesté à la lecture de cet article.

\section{Bibliographie}

Bachelard (G.), 1938. - La Psychanalyse du feu, Paris, Gallimard, 225 p.

Ballouche (A.), 2002. - « Histoire des paysages végétaux et mémoire des sociétés dans les savanes ouestafricaines », Historiens et géographes, vol. 381. p. 379-387.

Bassett (T. J.), Blanc Pamard (C.), Boutrais (J.), 2007. - « Constructing Locality: The Terroir Approach in West Africa », Africa, vol. 77, p. 104-129.

Benô̂t (M.), 1990. - « Gestion territoriale de l'activité agricole dans un village lorrain », Mappemonde, vol.4, p.15-17.

Berque (A.), 1992. - «Une certaine conception de l'environnement », dans Brunet (P.) (dir.), Atlas des Paysages ruraux de France, Paris, Edition de Monza, 200 p.

Blanc Pamard (C.), Milleville (P.), 1985. - « Pratiques paysannes, perception du milieu et système agraire », dans À travers champs. Agronomes et géographes. Dynamique des systèmes agraires, ORSTOM, Paris, 297 p.

Cadiou (N.), Luginbühl (Y.), 1995. - « Modèles paysagers et représentations du paysage en NormandieMaine », Paysage au pluriel: Pour une approche ethnologique des paysages, Editions de la Maison des Sciences de l'homme, Paris, p. 18-34.

Calvo-Iglesias (S.), Cressente-Maseda (R.), Fra-Pelo (U.), 2006. - « Exploring farmer's knowledge as a source of information on past and present cultural landscapes. A case study from NW Spain », Landscape and urban planning, vol.4, p. 334-343.

DarRe (J.-P.), 1985. - La parole et la technique. L'univers de pensée des agriculteurs du Ternois, L'Harmattan, Paris, $196 \mathrm{p}$. 
Darre (J.-P.), Mathieu (A.), Lasseur (J.), 2004. - Le sens des pratiques. Conceptions d'agriculteurs et modèles d'agronomes, Paris, INRA, coll. « Science update», 320 p.

Deffontaines (J.-P.), Petit (M.), 1985. - « Comment étudier les exploitations agricoles d'une région? Présentation d'un ensemble méthodologique », Etudes et recherches, n 4, 47 p.

Deffontaines (J.-P.), 1996. - « Du paysage comme moyen de connaissance de l'activité agricole à l'activité agricole comme moyen de production de paysage. L'agriculteur producteur de paysages. Un point de vue d'agronome », Comptes rendus de l'Académie d'agriculture de France, vol. 82, vol.4, p. 57-69.

—, 2001. - Les sentiers d’un géoagronome, éd. Arguments, Paris, 360 p.

—, 2004. - «L'objet dans l'espace agricole. Le regard d'un géo-agronome », Nature Sciences Sociétés, vol.12, p. 299-304.

De Sartre (X.-A.), Albaladejo (C.), Martins (P.), Veiga (I.), Grimaldi (M.), 2005. «Identification et évaluation de la diversité des modes d'exploitation des milieux en Amazonie orientale », Cahiers Agricultures, Vol. 14. p. 14-85.

Dugast (S.), 2008. «Incendies rituels et bois sacrés en Afrique de l'Ouest : une complémentarité méconnue », Bois et forêts des tropiques, vol. 296, p. 17-25.

Fairhead (J.), Leach (M.), 1996. Misreading the African Landscape: Society and Ecology in a Forest-Savanna Mosaic, Cambridge University Press, 232 p.

Friedberg (C.), 1992. « La question du déterminisme dans les rapports homme-nature », dans Jollivet (M.) (dir.), Sciences de la nature, sciences de la société : les passeurs de frontières, Paris, CNRS, p. 55-68.

Godelier (M.), 1984. L'idéel et le matériel, Paris, Fayard, 349 p.

Guisepelli (E.), 2005. «Les représentations sociales du paysage comme outils de connaissance préalable à l'action. L'exemple des Alpes du nord », Cybergeo : European Journal of Geography, 18 p. [http://www. cybergeo.eu/index3352.html].

Houet (T.), Corgne (S.), Hubert-Moy (L.), Marchand (J.-P.), 2008. «Approche systémique du fonctionnement d'un territoire bocager », L'espace géographique, $\mathrm{n}^{\circ}$ 3, p. 270-286.

Landais (E.), Deffontaines (J.-P.), Benoît (M.), 1988. « Les pratiques des agriculteurs. Point de vue sur un courant nouveau de la recherche agronomique », Etudes Rurales, vol.109, p. 125-158.

Le Floch (S.), Devanne (A.-S.), Deffontaines (J.-P.), 2005. « La fermeture du paysage: au-delà du phénomène, petite chronique d'une construction sociale », Espace Géographique n 1, p. 49-64.

Lemery (B.), 1986. «Usage des mots, présupposés des distinctions techniques / pratiques. Relations entre chercheurs et institutions de développement », Cahiers du GERDAL, n 11, p. 17-48.

Marie (M.), 2008. « Deux générations d'agriculteurs face aux transformations des paysages bocagers. Etude de cas en Normandie », Cahiers d'économie et de sociologie rurales, n 84-85, p. 192-214.

Marie (M.), Le Gouée (P.), Bermond (M.), 2008. « De la terre au sol : des logiques de représentations individuelles aux pratiques agricoles. Étude de cas en Pays d'Auge », Étude et gestion des sols, vol. 15, $\mathrm{n}^{\circ} 1$, p. 19-35.

Marie (M.), Delahaye (D.), 2009. « De la caractérisation des formes parcellaires aux usages agricoles de l'espace. Essai de comparaison des logiques d'organisation des paysages en domaine laitier et bocager en Europe de l'Ouest ", 9 Rencontres ThéoQuant, Besançon, 4-5-6 mars.

Mathieu (N.), 1992. "Géographie et interdisciplinarité : rapport naturel ou rapport interdit », dans Jollivet (M.) (dir.), Sciences de la nature, sciences de la société : les passeurs de frontières, Paris, CNRS, p. 129154.

Michelin (Y.), 1998. « Des appareils photo jetables au service d'un projet de développement : représentations paysagères et stratégies des acteurs locaux de la montagne thiernoise », Cybergéo : European Journal of Geography. [http://www.cybergeo.eu/index5351.html].

Pelissier (P.), 1980. «L'arbre en Afrique Tropicale, la fonction et le signe », cahiers ORSTOM-série sciences humaines, $\mathrm{n}^{\circ} 3-4$, p. 127-130.

Perichon (S.), 2004. « Les paysages campagnards sont-ils à l'image de ce que les agriculteurs souhaitent montrer d'eux-mêmes », Note de recherches de l'Académie d'Agriculture de France, 8 p. 
Sébastien Caillault, Maxime Marie

Peyre (D.), 2004. « La recherche, le développement, et les canards boîteux : quatre éleveurs face au même modèle de référence », dans Darre (J.-P.), Mathieu (A.) et Lasseur (J.), Le sens des pratiques. Conceptions d'agriculteurs et modèles d'agronomes, chap. 17, p. 237-253.

Poinsot (Y.), 2008. Comment l'agriculture fabrique ses paysages. Un regard géographique sur l'évolution des campagnes d'Europe, des Andes, et d'Afrique noire, Paris, Karthala, coll. « Hommes et Sociétés », 243 p.

Pourtier (R.), 2003. Les savanes africaines entre local et global : milieux, sociétés, espaces? Cahiers Agricultures, Vol. 12, n 4 , p. 213-218.

Prevel (M.), 2007. L'usine à la campagne. Une ethnographie du productivisme agricole, Paris, L'Harmattan, $299 \mathrm{p}$.

Pumain (D.), 2003. «Une approche de la complexité en géographie », Géocarrefour, vol. 18, n 1, p. 25-31.

Rossi (G.), 2000. L’ingérence écologique: environnement et développement rural du Nord au Sud : essai, Paris, CNRS, $248 \mathrm{p}$.

Sautter (G.), 1985. « Paysagismes », dans À travers champs, Agronomes et Géographes, Paris, ORSTOM, p. $289-297$.

Simon, (H.-A.), 1969. Les sciences de l'artificiel (traduit de l'anglais par J.-L. Le Moigne), Paris, Gallimard, $464 \mathrm{p}$.

Soulard (C.), 1999. Les agriculteurs et la pollution des eaux. Proposition d'une géographie des pratiques, Thèse de doctorat, Univ. Paris I, 425 p.

Serpantie (G.), Thomas (J.-N.), Douanio (M.), 2000. «Évolution contemporaine de la jachère dans les savanes cotonnières burkinabé », dans Floret (C.), Pontanier (R.), La jachère en Afrique tropicale: rôles, aménagement. Le point des connaissances, Édition John Libbey, vol. 1, p. 80-91.

TALlET (B.), 2007. À l'arrière des fronts pionniers : recompositions territoriales dans l'Ouest du Burkina Faso et le Sud du Veracruz, Habilitation à diriger les recherches, Tome 3, Paris I, 206 p.

Tersiguel (P.), 1995. Le pari du tracteur : la modernisation de l'agriculture cotonnière au Burkina Faso, Paris, Edition Orstom, 284 p.

Thenall (C.), 1996. Exploitations agricoles et territoire(s) : contribution à la structuration de la mosaïque paysagère, Thèse de doctorat, Univ. Rennes I, 379 p.

Veauvy (C.), 1991. "Usure des sols et représentations sociales. Le cas de la Provence contemporaine », dans Savoirs paysans et développement, Paris, Karthala-Orstom, p. 111-133.

Cet article a été reçu le $1^{\text {er }}$ mars 2009 et définitivement accepté le 10 juin 2009. 
planche I (Sébastien CAILLAULT, Maxime MARIE - Pratiques agricoles, perceptions et représentations du paysage...)

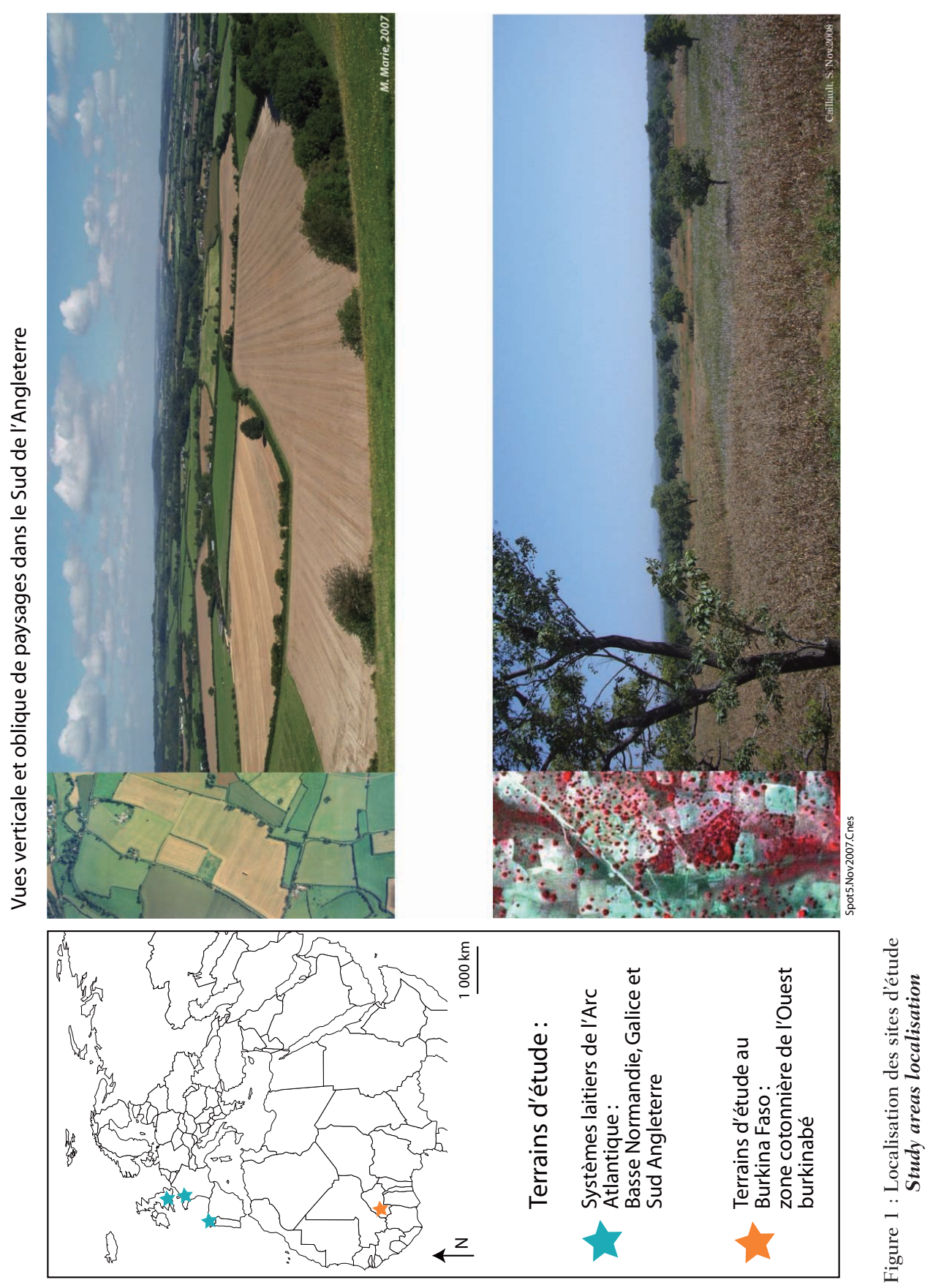


planche II (Sébastien CAILLAULT, Maxime MARIE - Pratiques agricoles, perceptions et représentations du paysage...)

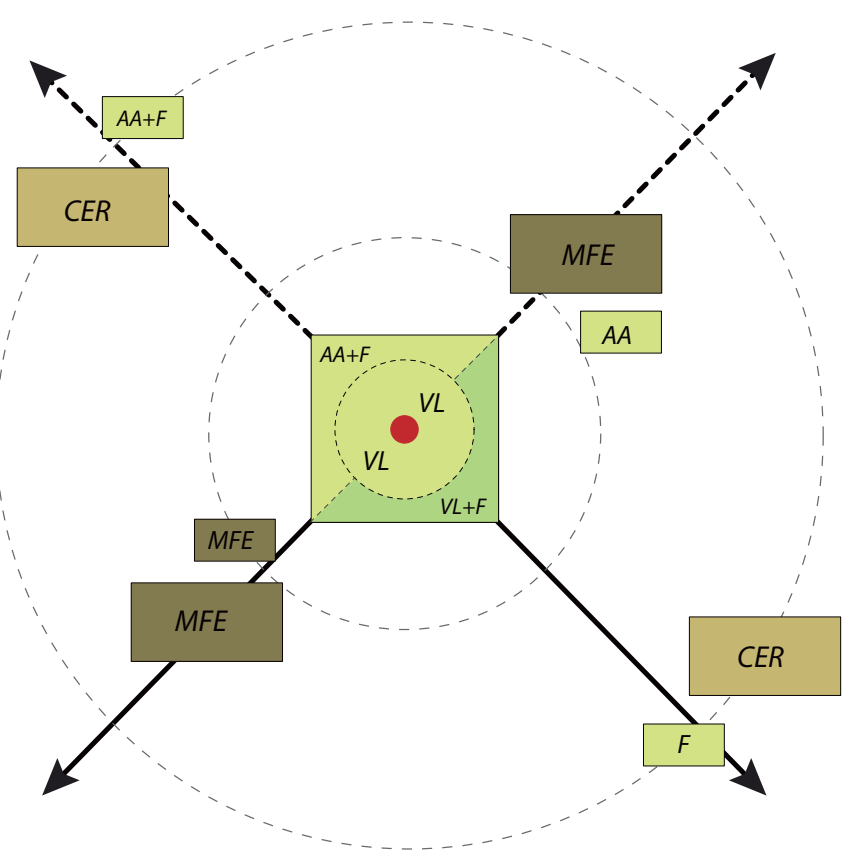

\section{Légende :}

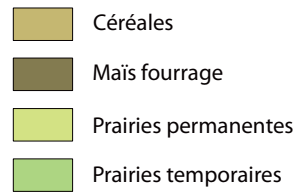

\section{Abréviations :}

$V L$ : pâtures Vaches Laitières

$A A$ : pâtures Autres Animaux (Génisses, VA) $V L+F$ : pâtures Vaches Laitières + Fauche $A A+F$ : pâtures Autres Animaux + Fauche $F$ : prairies de Fauche (ensilage ou foin) $M F E$ : rotations à dominante Maïs Fourrage CER: rotations à dominante Céréales

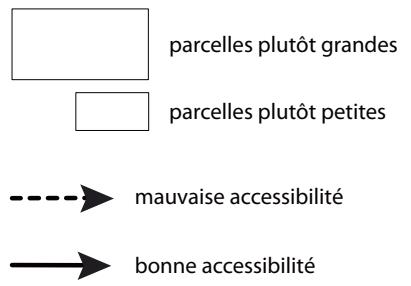

Figure 2 : Modèle d'organisation des exploitations laitières anglaises Organizational model of dairy farms in southern England
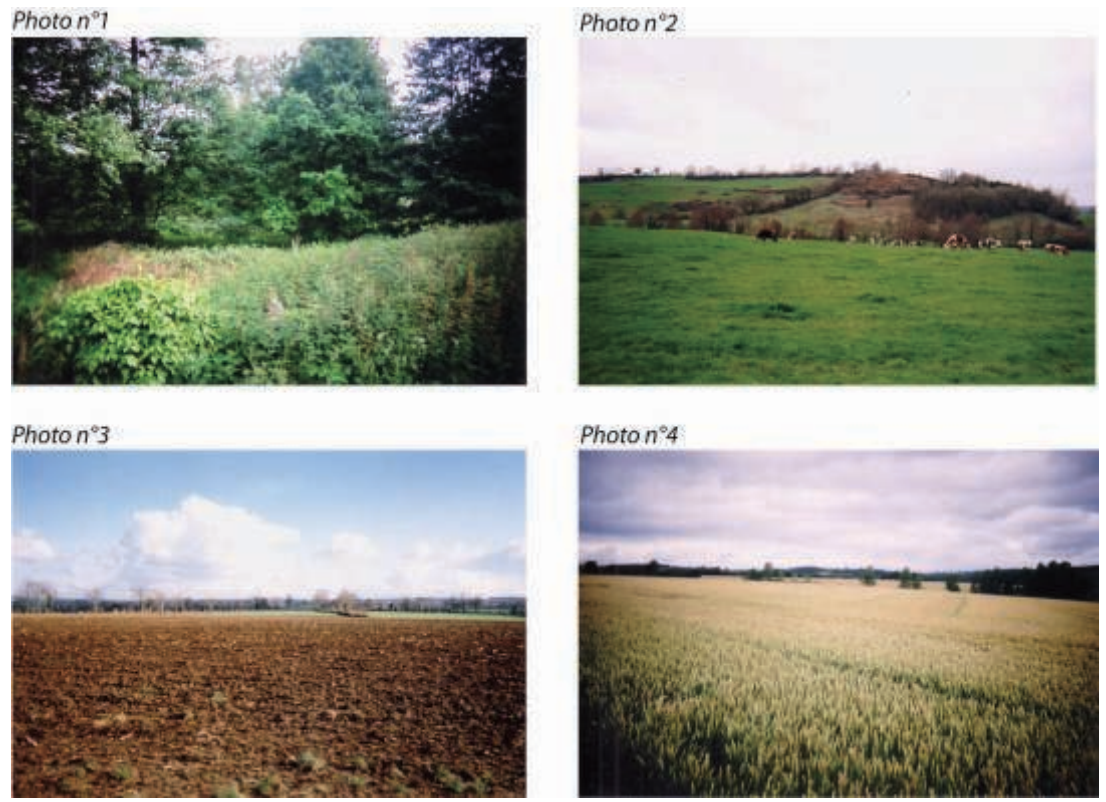

Figure 3 : Les représentations paysagères des agriculteurs Landscape representations of farmers 
planche III (Sébastien CAILLAULT, Maxime MARIE - Pratiques agricoles, perceptions et représentations du paysage...)
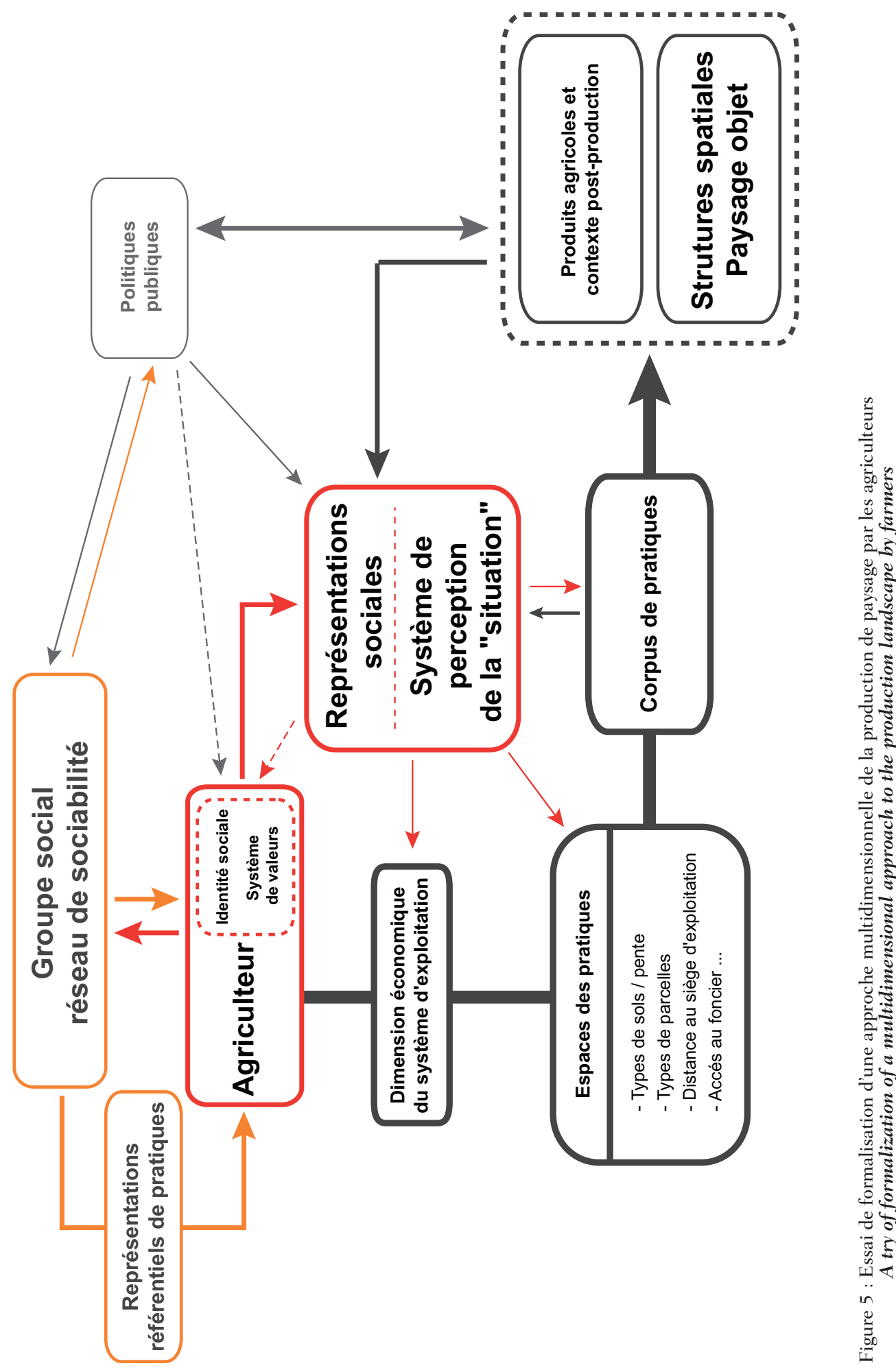